\title{
Clinical application of biological markers for treatments of resectable non-small-cell lung cancers
}

\author{
C Huang*,', D Liu', D Masuya', T Nakashima', K Kameyama', S Ishikawa', M Ueno², R Haba ${ }^{3}$ and H Yokomise' \\ 'Second Department Surgery, Faculty of Medicine, Kagawa University, 1 750-1, Miki-cho, Kita-gun, Kagawa 76 I-0793, Japan; ${ }^{2}$ Pathology and Host \\ Defense, Faculty of Medicine, Kagawa University, Kagawa, Japan; ${ }^{3}$ Department of Pathology, Faculty of Medicine, Kagawa University, Kagawa, Japan
}

We performed a clinical study to identify biological markers useful for the treatment of resectable non-small-cell lung cancers (NSCLCs). In all, I 73 patients were studied. By immunohistochemistry, we evaluated the Ki-67 proliferation index, tumour vascularity, thymidylate synthase (TS), vascular endothelial growth factor (VEGF)-A, VEGF-C, and E (epithelial)-cadherin. Concerning the survival of NSCLC patients, tumour vascularity $(P<0.01)$, VEGF-A status $(P=0.03)$, VEGF-C status $(P=0.03)$, and E-cadherin status $(P=0.03)$ were significant prognostic factors in patients with stage I NSCLCs. The Ki-67 proliferation index $(P=0.02)$ and TS status $(P<0.0$ I) were significant prognostic factors in patients with stage II-III NSCLCs. In patients with stage II-III NSCLCs, furthermore, the survival of UFT (a combination of tegafur and uracil)-treated patients with TS-negative tumours was significantly better than those of any other patients. Biological markers associated with tumour angiogenesis or metastasis are useful for the detection of aggressive tumours among early-stage NSCLCs. Postoperative chemotherapy might be necessary in such tumours even in stage I. In contrast, tumour proliferation rate and TS status are useful markers for identifying less aggressive tumours in locally advanced NSCLCs. Thymidylate synthase expression is also a useful marker to evaluate responsiveness of UFT-based chemotherapy for these tumours. British Journal of Cancer (2005) 92, I231-1239. doi:I0.1038/sj.bjc.660248I www.bjcancer.com

Published online 22 March 2005

(c) 2005 Cancer Research UK

Keywords: lung cancer; biological marker; prognosis; immunohistochemistry

Non-small-cell lung cancer (NSCLC) is one of the most common human malignancies with a poor prognosis. Surgical resections play major roles in managing patients with stage I and stage II NSCLCs, and may be used for patients with stage III NSCLCs (Cersosimo, 2002). In addition, various combined-modality therapy, including chemotherapy and radiation therapy, have been assessed for improving the outcome of patients with NSCLCs (Arriagada et al, 2004; Machtay et al, 2004; Strauss et al, 2004; Winton et al, 2004). However, the 3-year survival of stage II NSCLC patients was 35-55\%, and the 3-year survival of resectable stage III NSCLC patients was $28 \%$ (Martin et al, 2002). Therefore, a new therapeutic strategy is required for patients with resectable NSCLCs.

Owing to developments in molecular biology, many clinical studies on molecular markers associated with tumour biological behaviour have been performed in human cancers, and these molecular markers, including oncogenes, tumour suppressor genes (Huang et al, 1998), metastatic suppressor genes (Adachi et al, 1998; Sulzer et al, 1998), and angiogenetic factors (Imoto et al, 1998; Masuya et al, 2001), could be prognostic factors for NSCLC patients. Therefore, to improve the treatment of NSCLC patients, it is important to decide the optimal therapeutic strategy for them according to the tumour biology. Our previous studies demonstrated four biological markers, including thymidylate synthase (TS) (Huang et al, 2000), vascular endothelial growth factor-A

*Correspondence: Dr C Huang; E-mail: chuang@kms.ac.jp

Received 16 August 2004; revised 20 January 2005; accepted 31 January 2005; published online 22 March 2005
(VEGF-A), VEGF-C (Nakashima et al, 2004), and E (epithelial)cadherin (Liu et al, 2001), to be significant prognostic factors for NSCLC patients. Thymidylate synthase is reported to be related to tumour cell proliferation and responsiveness to 5-FU-based chemotherapy (Navalgund et al, 1980; Beck et al, 1994). VEGF-A and VEGF-C are members of the VEGF family associated with angiogenesis or lymphangiogenesis (Dvorak et al, 1995; Skobe et al, 2001). E-cadherin is one of the metastatic suppressor genes (Mbalaviele et al, 1996; Sulzer et al, 1998).

Therefore, we performed a retrospective clinical study to identify molecular markers useful for the treatment of patients with resectable NSCLCs. We evaluated the intratumoral expressions of these four biological markers: TS, VEGF-A, VEGF-C, and E-cadherin. In addition, we studied the tumour proliferation rate using the Ki-67 index (Gerdes et al, 1984; Scagliotti et al, 1993) and tumour angiogenesis using CD34 staining (Matsuyama et al, 1998).

\section{MATERIALS AND METHODS}

\section{Clinical characteristics of patients}

From January 1995 to December 1997, consecutive NSCLC patients who underwent surgery at the Second Department of Surgery, Faculty of Medicine, Kagawa University were studied. This study was approved by the institutional review board of Kagawa University (14-7, a clinical study of biological markers in NSCLCs). TNM staging designations were made according to the postsurgical pathological international staging system (Mountain, 1997). In total, 173 patients with lung cancer up to stage III were 
investigated (Table 1). These included 97 patients with stage I NSCLCs, 17 patients with stage II NSCLCs, and 59 patients with stage III NSCLCs. Patients' clinical records and histopathological diagnoses were fully documented. This report includes follow-up data as of 31 March 2004. The median follow-up period for all patients was $77.0 \pm 28.9$ months.

Regarding methods of surgical resection, a pneumonectomy was performed in 16 patients with stage II-III NSCLCs. A lobectomy was performed in 133 patients: 73 patients with stage I NSCLCs, 15 patients with stage II NSCLCs, and 45 patients with stage III NSCLCs. A segmentectomy was performed in three patients with stage I NSCLCs, and a wedge resection was performed in 21 patients with stage I NSCLCs. Systemic chemotherapy using mitomycin/vinblastin/cisplatin (MVP) was performed in all patients with stage II-III NSCLCs; neoadjuvant chemotherapy in 49 patients, and postoperative adjuvant chemotherapy in 27 patients with nodal metastases. Radiation therapy was performed in 18 patients: 11 patients with $\mathrm{T} 3$ or T4 status and seven patients with mediastinal lymph node metastases. 5-FU was administered as UFT (a combination of tegafur and uracil, Taiho Pharmaceutical Co., Tokyo, Japan; $300-400 \mathrm{mg} \mathrm{day}^{-1}$ body $^{-1}$ ) to 107 patients

Table I Patient demographics

\begin{tabular}{|c|c|c|}
\hline Patient characteristics & Number & (\%) \\
\hline Total number of patients & 173 & 100 \\
\hline \multicolumn{3}{|l|}{ Age (years) } \\
\hline Median & 67 & \\
\hline Range & $35-76$ & \\
\hline \multicolumn{3}{|l|}{ Gender } \\
\hline Male & 116 & 67 \\
\hline Female & 57 & 33 \\
\hline \multicolumn{3}{|l|}{ Smoking habits } \\
\hline Nonsmoker & 65 & 38 \\
\hline Smoker & 108 & 62 \\
\hline \multicolumn{3}{|l|}{ ECOG performance status } \\
\hline 0 & 83 & 48 \\
\hline I & 74 & 43 \\
\hline 2 & 16 & 9 \\
\hline \multicolumn{3}{|l|}{ Histology } \\
\hline Adenocarcinoma & 101 & 58 \\
\hline Squamous cell carcinoma & 58 & 34 \\
\hline Large-cell carcinoma & 14 & 8 \\
\hline \multicolumn{3}{|l|}{ Pathological stage } \\
\hline I & 97 & 56 \\
\hline ॥ & 17 & 10 \\
\hline III & 59 & 34 \\
\hline \multicolumn{3}{|l|}{ Method of surgical resection } \\
\hline Pneumonectomy & 16 & 9 \\
\hline Lobectomy & 133 & 77 \\
\hline Segmentectomy & 3 & 2 \\
\hline Wedge resection & 21 & 12 \\
\hline \multicolumn{3}{|l|}{ Chemotherapy } \\
\hline MVP & 76 & 44 \\
\hline Neoadjuvant therapy & 49 & 28 \\
\hline Postoperative adjuvant therapy & 27 & 16 \\
\hline UFT & 107 & 62 \\
\hline Radiotherapy & 18 & 10 \\
\hline
\end{tabular}

ECOG = Eastern Cooperative Oncology Group; MVP = mitomycin/vinblastin/cisplatin; UFT = a combination of tegafur and uracil. including 53 patients with stage I NSCLCs and 54 patients with stage II-III NSCLCs. Oral administration of UFT was started within 1 month after surgery. UFT was administered for 2 years if there was no recurrence of carcinomas. In patients with recurrences, UFT was given until its oral administration became impossible.

\section{Immunohistochemistry}

The following antibodies were used, along with isotype antibodies as negative controls: a mouse monoclonal antibody for the Ki-67 antigen (MIB-1, DAKO, Glostrup, Denmark) diluted at 1:40, a mouse monoclonal antibody for CD34 (NU-4A1, Nichirei Corporation, Tokyo, Japan) diluted at $1: 10$, a rabbit polyclonal antibody for TS (kindly provided by Dr M Fukushima) (Huang et al, 2000) diluted at 1:500, a rabbit polyclonal antibody for VEGF-A (A-20, Santa Cruz Biotechnology, Santa Cruz, CA, USA) diluted at $1: 200$, a goat polyclonal antibody for VEGF-C (N-19, Santa Cruz Biotechnology) diluted at $1: 100$, and a mouse monoclonal antibody for E-cadherin (HECD-1, Takara, Otsu, Japan) diluted at $1: 400$.

Formalin-fixed paraffin-embedded tissue was cut into $4-\mu \mathrm{m}$ sections and mounted onto poly-L-lysine-coated slides. Sections were deparaffinised and rehydrated. The slides were then heated in a microwave for $10 \mathrm{~min}$ in a $10-\mu \mathrm{moll}^{-1}$ citrate buffer solution at $\mathrm{pH} 6.0$, and cooled to room temperature for $20 \mathrm{~min}$. After quenching the endogenous peroxidase activity with $0.3 \% \mathrm{H}_{2} \mathrm{O}_{2}$ (in absolute methanol) for $30 \mathrm{~min}$, the sections were treated for $2 \mathrm{~h}$ at room temperature with $5 \%$ bovine serum albumin to block nonspecific staining. Duplicate sections were incubated overnight with the primary specific antibodies detecting $\mathrm{Ki}-67, \mathrm{CD} 34$, TS, VEGF-A, VEGF-C, and E-cadherin. Slides were then incubated for $1 \mathrm{~h}$ with biotinylated anti-mouse IgG (Vector Laboratories Inc., Burlingame, CA, USA) for Ki-67, CD34, and E-cadherin, biotinylated anti-rabbit IgG (Vector Laboratories Inc.) for TS and VEGF$A$, and biotinylated anti-goat IgG (Vector Laboratories Inc.) for VEGF-C. The sections were incubated with the avidin-biotinperoxidase complex (Vector Laboratories Inc.) for $1 \mathrm{~h}$, and antibody binding was visualised with $3,3^{\prime}$-diaminobenzidine tetrahydrochloride. Lastly, the sections were lightly counterstained with Mayer's haematoxylin. The human colon cancer cell line DLD-1/FrUrd was used as a positive control for the staining of TS. Sections of resected lung tumours known to express VEGF-A, VEGF-C, or E-cadherin were used as positive controls for immunohistochemic staining, respectively.

\section{Assessment of immunohistochemical staining}

All of the immunostained sections were reviewed by two pathologists (M Ueno and $\mathrm{R}$ Haba) who had no knowledge of the patients' clinical status. The percentage of carcinoma cells with positive staining for $\mathrm{Ki}-67$ in a given specimen was scored as the Ki-67 proliferation index, and tumours with $\geqslant 25 \%$ of $\mathrm{Ki}-67$ proliferation index were classified as high Ki-67 (Scagliotti et al, 1993). For microvessel quantification, the three most highly vascularised areas detected by CD34 immunostaining were initially selected under the $\times 40$ field, and a $\times 200$ field $\left(0.785 \mathrm{~mm}^{2}\right.$ per field) was used to count vessels in each of these areas. The average of three $\times 200$ field counts was recorded as the intratumoral microvessel density (IMD). Tumours with $\geqslant 90$ of the IMD were classified as hypervascular (Masuya et al, 2001).

For TS, VEGF-A VEGF-C, and E-cadherin, samples were classified into two groups, positive or negative, with a cutoff value based on the findings of previous reports, respectively. At least 200 tumour cells were scored per $\times 40$ field. All sections were scored in a semiquantitative manner according to the method described previously, which reflects both the intensity and percentage of cells staining at each intensity (McCarty et al, 1986). Intensity was 
classified as 0 (no staining), +1 (weak staining), +2 (distinct staining), or +3 (very strong staining). A value designated the 'HSCORE' was obtained for each slide by using the following algorithm: HSCORE $=\Sigma(I \times \mathrm{PC})$, where $I$ and PC represent staining intensity and the percentage of cells that stain at each intensity, respectively, and the corresponding HSCOREs were calculated separately. Concerning TS expression, when the HSCORE of TS in a given specimen was $\geqslant 30$, the sample was classified as TS-positive (Huang et al, 2000). Expressions of VEGF$\mathrm{A}, \mathrm{VEGF}-\mathrm{C}$, and E-cadherin were classified as follows: when $\geqslant 30 \%$ of the carcinoma cells in a given specimen were positively stained for VEGF-A, the sample was classified as VEGF-A-positive (Masuya et al, 2001); when $\geqslant 30 \%$ of the carcinoma cells in a given specimen were positively stained for VEGF-C, the sample was classified as VEGF-C-positive (Arinaga et al, 2003); and when $\geqslant 50 \%$ of the carcinoma cells in a given specimen were positively stained for E-cadherin, the sample was classified as E-cadherinpositive (Liu et al, 2001).

\section{Statistical analysis}

The statistical differences in the expression of each biological marker in relation to various clinical and pathological parameters were assessed by the $\chi^{2}$ test. Overall survival was defined as the time from treatment initiation (surgical resection, chemotherapy, or radiation) to the date of death from any cause. The KaplanMeier method was used to estimate the probability of overall survival as a function of time, and differences in the survival of subgroups of patients were compared by using Mantel's log-rank test. Multivariate analyses were performed using the Cox regression model to study the effects on survival (Cox, 1972). The validity of the proportional hazards assumption was investigated by graphical methods (Grambsch and Therneau, 1994). All Pvalues were based on two-tailed statistical analysis, and a $P$-value $<0.05$ was considered to indicate statistical significance.

\section{RESULTS}

\section{Distribution of biological markers in NSCLCs}

Concerning the Ki-67 proliferation index, 117 carcinomas (67.6\%) had high Ki-67 (Table 2). The frequency of high Ki-67 tumours was significantly higher in squamous cell carcinomas than in adenocarcinomas $(87.9$ vs $56.4 \%, P<0.01)$. However, there was no difference in the Ki-67 index in relation to tumour status, nodal status, pathological stage, or neoadjuvant chemotherapy.

With respect to tumour angiogenesis, 93 carcinomas $(53.8 \%)$ were hypervascular tumours. The frequency of hypervascular tumours was significantly higher in adenocarcinomas than in squamous cell carcinomas ( 65.3 vs $32.8 \%, P<0.01)$. There was no difference in the IMD according to tumour status, nodal status, pathological stage, or neoadjuvant chemotherapy.

Concerning the intratumoral TS expression status, 93 carcinomas $(53.8 \%)$ were TS-positive. The frequency of TS-positive tumours was significantly higher in squamous cell carcinomas than in adenocarcinomas ( 72.4 vs $43.6 \%, P<0.01)$. There was also no difference in TS status according to tumour status, nodal status, pathological stage, or neoadjuvant chemotherapy.

In all, 91 carcinomas (52.6\%) were VEGF-A-positive tumours. There was no difference in VEGF-A status in relation to tumour histology or neoadjuvant chemotherapy. However, the frequency of nodal metastases was significantly higher in VEGF-A-positive tumours than in VEGF-A-negative tumours (37.4 vs 23.2\%, $P=0.04)$.

A total of 73 carcinomas $(42.2 \%)$ were VEGF-C-positive tumours. There was no difference in VEGF-C status in relation to tumour histology, tumour status, nodal status, pathological stage, or neoadjuvant chemotherapy.

In total, 98 carcinomas (56.6\%) were E-cadherin-negative. There was no difference in E-cadherin status in relation to tumour histology or neoadjuvant chemotherapy. However, the frequency

Table 2 Distribution of biological markers in 173 NSCLC patients according to clinicopathological characteristics

\begin{tabular}{|c|c|c|c|c|c|c|c|c|c|c|c|c|c|}
\hline \multirow[b]{2}{*}{ Characteristics } & \multirow[b]{2}{*}{$n$} & \multicolumn{2}{|c|}{ High Ki-67 } & \multicolumn{2}{|c|}{ Hypervascular } & \multicolumn{2}{|c|}{ TS-positive } & \multicolumn{2}{|c|}{ VEGF-A-positive } & \multicolumn{2}{|c|}{ VEGF-C-positive } & \multicolumn{2}{|c|}{ E-cadherin-negative } \\
\hline & & (\%) & $P$-value & (\%) & $P$-value & (\%) & $P$-value & (\%) & $P$-value & (\%) & $P$-value & (\%) & $P$-value \\
\hline \multicolumn{14}{|l|}{ Smoking } \\
\hline Nonsmoker & 65 & 50.8 & $<0.01$ & 61.5 & 0.15 & 53.8 & 0.98 & 49.2 & 0.49 & 38.5 & 0.44 & 55.4 & 0.79 \\
\hline Smoker & 108 & 77.8 & & 49.1 & & 53.7 & & 54.6 & & 44.4 & & 57.4 & \\
\hline \multicolumn{14}{|l|}{ Tumour status } \\
\hline $\mathrm{T} 1, \mathrm{~T} 2$ & 125 & 68.8 & 0.59 & 52.0 & 0.45 & 50.4 & 0.15 & 51.2 & 0.55 & 41.6 & 0.79 & 53.6 & 0.19 \\
\hline $\mathrm{T} 3, \mathrm{~T} 4$ & 48 & 64.6 & & 58.3 & & 62.5 & & 56.3 & & 43.8 & & 64.6 & \\
\hline \multicolumn{14}{|l|}{ Nodal status } \\
\hline NO & 120 & 65.0 & 0.26 & 53.3 & 0.86 & 51.7 & 0.40 & 47.5 & 0.04 & 42.5 & 0.90 & 50.8 & 0.02 \\
\hline $\mathrm{N} 1, \mathrm{~N} 2$ & 53 & 73.6 & & 54.7 & & 58.5 & & 64.2 & & 41.5 & & 69.8 & \\
\hline \multicolumn{14}{|l|}{ Pathological status } \\
\hline Stage I & 97 & 69.1 & 0.39 & 51.5 & 0.54 & 52.6 & 0.39 & 48.5 & 0.21 & 40.2 & 0.78 & 50.5 & 0.09 \\
\hline Stage ॥ & 17 & 52.9 & & 47.1 & & 41.2 & & 29.4 & & 41.2 & & 76.5 & \\
\hline Stage III & 59 & 69.5 & & 59.3 & & 59.3 & & 66.1 & & 45.8 & & 61.0 & \\
\hline \multicolumn{14}{|l|}{ Histology } \\
\hline Adenocarcinoma & $10 \mid$ & 56.4 & $<0.01$ & 65.3 & $<0.01$ & 43.6 & $<0.01$ & 54.5 & 0.70 & 45.5 & 0.22 & 52.5 & 0.06 \\
\hline Squamous cell carcinoma & 58 & 87.9 & & 32.8 & & 72.4 & & 51.7 & & 41.4 & & 56.9 & \\
\hline Large-cell carcinoma & 14 & 64.3 & & 57.1 & & 50.0 & & 42.9 & & 21.4 & & 85.7 & \\
\hline \multicolumn{14}{|c|}{ Neoadjuvant MVP chemotherapy } \\
\hline Without neoadjuvant MVP & 124 & 70.9 & 0.19 & 52.4 & 0.69 & 53.2 & 0.95 & 52.4 & 0.93 & 43.5 & 0.68 & 54.0 & 0.35 \\
\hline With neoadjuvant MVP & 49 & 59.2 & & 57.1 & & 55.1 & & 53.1 & & 38.8 & & 63.3 & \\
\hline Total number of patients & 173 & 67.6 & & 53.8 & & 53.8 & & 52.6 & & 42.2 & & 56.6 & \\
\hline
\end{tabular}

NSCLC = non-small-cell lung cancer; TS = thymidylate synthase; VEGF = vascular endothelial growth factor; MVP=mitomycin/vinblastin/cisplatin 
of nodal metastases was significantly higher in E-cadherin-negative tumours than in E-cadherin-positive tumours (37.8 vs 21.3\%, $P=0.02)$.

\section{Ki-67 proliferation index in relation to biological markers in NSCLCs}

Concerning TS status, the Ki-67 proliferation index was significantly higher in TS-positive tumours than in TS-negative tumours $(48.6 \pm 29.9$ vs $35.6 \pm 29.8, P<0.01)$. Regarding VEGF-A status, the Ki-67 proliferation index was significantly higher in VEGF-Apositive tumours than in VEGF-A-negative tumours $(46.9 \pm 31.5 \mathrm{vs}$ $37.7 \pm 28.6, P=0.04)$. However, there was no difference in the $\mathrm{Ki}$ 67 proliferation index in relation to VEGF-C or E-cadherin status.

\section{IMD in relation to biological markers in NSCLCs}

Concerning VEGF-A status, the IMD was significantly higher in VEGF-A-positive tumours than in VEGF-A-negative tumours $(118.9 \pm 51.6$ vs $91.1 \pm 55.8, \quad P<0.01)$. However, there was no difference in the IMD in relation to TS, VEGF-C, or E-cadherin status.

\section{Survival of patients with stage I NSCLCs in relation to biological markers}

The 5-year survival rates of 97 patients with stage I NSCLCs in relation to biological markers are shown in Table 3 . With respect to patients with stage I NSCLCs, there was no significant difference in the 5-year survival rates between patients with high $\mathrm{Ki}-67$ tumours and those with low Ki-67 tumours (70.0 vs $82.2 \%$, Figure 1A). In contrast, the 5-year survival rate of patients with hypervascular tumours was significantly lower than that of patients with hypovascular tumours (58.3 vs 83.5\%, $P<0.01$, Figure 1B).

On the other hand, there was no significant difference in the 5year survival rates between patients with TS-positive tumours and those with TS-negative tumours (60.7 vs 81.8\%, Figure 1C).
Regarding the TS status and UFT-based chemotherapy, there was no significant difference in the 5-year survival rates according to intratumoral TS status and UFT-based chemotherapy among stage I NSCLCs (Figure 1D). There was also no significant difference in the 5-year survival rates according to intratumoral TS status and UFT-based chemotherapy among stage I adenocarcinomas of the lung $(72.2 \%$ in UFT-treated patients with TS-negative tumours, $77.2 \%$ in UFT-untreated patients with TS-negative tumours, $56.3 \%$ in UFT-treated patients with TS-positive tumours, and $66.7 \%$ in UFT-untreated patients with TS-positive tumours).

In contrast, the 5-year survival rate of patients with VEGF-Apositive tumours was significantly lower than that of patients with VEGF-A-negative tumours (58.6 vs $82.2 \%, P=0.01$, Figure $1 \mathrm{E})$. The 5-year survival rate of patients with VEGF-C-positive tumours was also significantly lower than that of patients with VEGF-Cnegative tumours ( 54.9 vs $80.3 \%, P<0.01$, Figure $1 \mathrm{~F}$ ). Furthermore, the 5-year survival rate of patients with E-cadherin-negative tumours was significantly lower than that of patients with Ecadherin-positive tumours ( 55.2 vs $85.3 \%, P=0.01$, Figure $1 G$ ).

The multivariate analysis demonstrated that four variables, IMD (hazard ratio $=3.34, P<0.01$ ), VEGF-A status (hazard ratio $=2.37$, $P=0.03$ ), VEGF-C status (hazard ratio $=2.10, P=0.03$ ), and $\mathrm{E}$ cadherin status (hazard ratio $=2.30, P=0.03$ ), were significant prognostic factors in patients with stage I NSCLCs (Table 4). The proportional hazards assumption was met adequately.

\section{Survival of patients with stage II-III NSCLCs in relation to biological markers}

The 5-year survival rates of 76 patients with stage II - III NSCLCs in relation to biological markers are also shown in Table 3. With respect to patients with stage II-III (locally advanced) NSCLCs, the 5-year survival rate of patients with low Ki-67 tumours was significantly higher than that of patients with high Ki-67 tumours (43.2 vs $20.0 \%, P=0.02$, Figure $2 \mathrm{~A}$ ). Especially, the 5-year survival rate of patients with low Ki-67 tumours was significantly higher than that of patients with high Ki-67 tumours among stage III (42.8

Table 3 The 5-year survival rate of I73 NSCLC patients in relation to biological markers and pathological stage

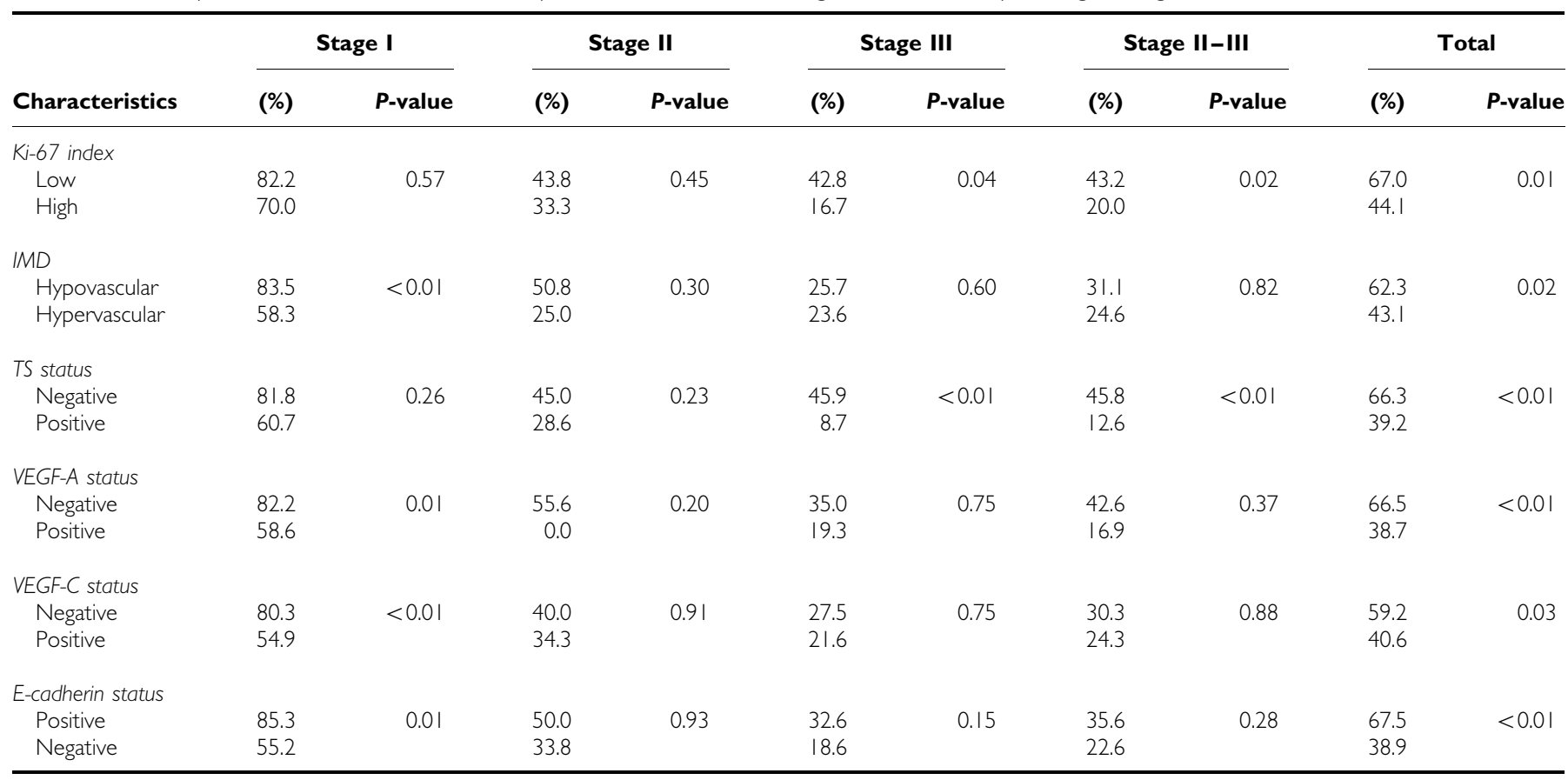

NSCLC = non-small-cell lung cancer; IMD = intratumoral microvessel density; TS=thymidylate synthase; VEGF=vascular endothelial growth factor; MVP= mitomycin/ vinblastin/cisplatin; E-cadherin = epithelial cadherin. 


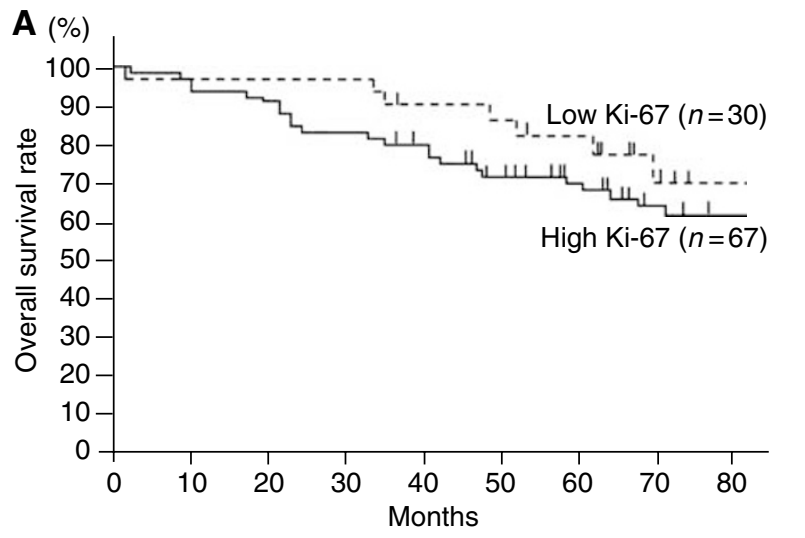

B (\%)
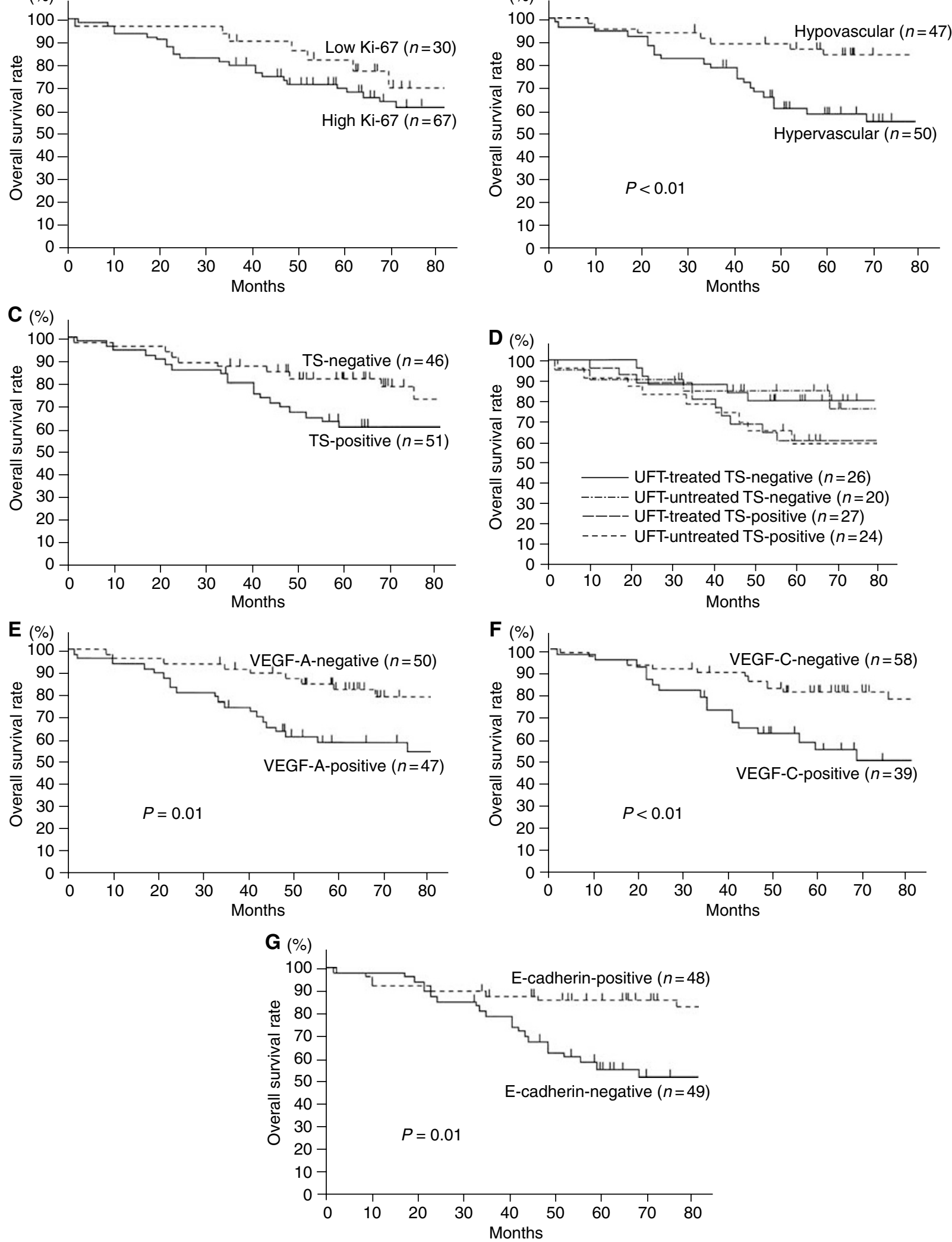

Figure I Overall survival of patients with stage I NSCLCs in relation to biological markers. (A) Ki-67 index, (B) tumour vascularity, (C) TS status, (D) TS status and UFT-based chemotherapy, (E) VEGF-A status, (F) VEGF-C status, and (G) E-cadherin status.

vs $16.7 \%, P=0.04)$. However, there was no difference in the 5 -year survival rates between patients with hypovascular tumours and those with hypervascular tumours (31.1 vs $24.6 \%$, Figure $2 \mathrm{~B})$.
Concerning TS status, the 5-year survival rate of patients with TS-negative tumours was significantly higher than that of patients with TS-positive tumours among stage II-III (45.8 vs $12.6 \%$, 
Table 4 Multivariate regression analyses in predicting survival of NSCLC patients

\begin{tabular}{|c|c|c|c|c|c|c|}
\hline Biological markers & \multicolumn{3}{|c|}{ Stage I } & \multicolumn{3}{|c|}{ Stage II, Stage III } \\
\hline IMD & 3.34 & $1.30-7.86$ & $<0.01$ & 1.38 & $0.75-2.54$ & 0.28 \\
\hline TS status & 1.31 & $0.57-2.98$ & 0.51 & 2.43 & $1.34-4.42$ & $<0.01$ \\
\hline VEGF-A status & 2.37 & $1.07-5.24$ & 0.03 & 1.27 & $0.69-2.35$ & 0.43 \\
\hline VEGF-C status & 2.10 & $1.01-4.37$ & 0.03 & 0.94 & $0.54-1.64$ & 0.83 \\
\hline
\end{tabular}

NSCLC = non-small-cell lung cancer; $C I=$ confidence interval; IMD = intratumoral microvessel density; TS = thymidylate synthase; VEGF = vascular endothelial growth factor; $\mathrm{MVP}=$ mitomycin/vinblastin/cisplatin; E-cadherin = epithelial cadherin.

$P<0.01$, Figure 2C). Especially, the 5-year survival rate of patients with TS-negative tumours was significantly higher than that of patients with TS-positive tumours among stage III (45.9 vs 8.7\%, $P<0.01)$. Regarding TS status and UFT-based chemotherapy, furthermore, the 5-year survival rates were $53.8 \%$ in UFT-treated patients with TS-negative tumours, $15.0 \%$ in UFT-untreated patients with TS-negative tumours, $15.2 \%$ in UFT-treated patients with TS-positive tumours, and $7.1 \%$ in UFT-untreated patients with TS-positive tumours (Figure 2D). In patients with stage II-III NSCLCs, the 5-year survival rate of UFT-treated patients with TSnegative tumours was significantly higher than those of any other patients $(P=0.04, P<0.01$, and $P<0.01$, respectively).

However, there was no difference in the survival of patients with stage II-III NSCLCs in relation to intratumoral expressions of VEGF-A, VEGF-C, or E-cadherin (Figure 2E-G).

The multivariate analysis also demonstrated that the Ki-67 proliferation index (hazard ratio $=2.04, P=0.02$ ) and TS status (hazard ratio $=2.43, P<0.01$ ) were significant prognostic factors in patients with stage II-III NSCLCs (Table 4). The proportional hazards assumption was met adequately.

\section{DISCUSSION}

Recent studies on molecular biology in human cancers have revealed that many molecules affect various biological behaviours of malignant tumours. For example, the activation of oncogenes or the inactivation of tumour suppressor genes, such as K-ras mutation and p53 mutation, could initially cause malignant progression (Huang et al, 1998). In addition, reduced expressions of metastatic suppressor genes, such as E-cadherin (Sulzer et al, 1998; Liu et al, 2001), MRP-1/CD9, and KAI1/CD82 (Adachi et al, 1998 ), could induce tumour cells with high metastatic potential. In addition, tumour angiogenesis, affected by various angiogenetic factors such as the VEGF family (Dvorak et al, 1995), is associated with not only tumour growth but also tumour metastasis (Folkman, 1995).

The clinical use of these biological markers could be classified into categories as follows: detection of aggressive tumours with a poor prognosis among early stage tumours (Adachi et al, 1998; Liu et al, 2001), identification of less aggressive tumours with a relatively good prognosis among advanced stage tumours, and selection of effective adjuvant therapies including chemotherapy (made-to-order medicine) (Rosell et al, 2003; Vokes and Choy, 2003).

Many biological markers have been reported to be correlated with the survival of NSCLC patients. However, most biological markers associated with tumour angiogenesis and metastatic potential are correlated with the prognosis of early-stage NSCLCs (Adachi et al, 1998; Liu et al, 2001; Masuya et al, 2001). The present study also demonstrated that the survival of patients with stage I NSCLCs was significantly lower in patients with hypervascular tumours than in patients with hypovascular tumours. In addition, VEGF-C expression, another member of the VEGF family and reported to induce lymphangiogenesis (Skobe et al, 2001), was also associated with the prognosis of stage I patients, as reported previously (Kajita et al, 2001; Arinaga et al, 2003). Furthermore, previous clinical studies have shown that reduced expressions of several metastatic suppressor genes, including E-cadherin (Sulzer et al, 1998; Liu et al, 2001), MRP-1/CD9, and KAI1/CD82 (Adachi et al, 1998), are also associated with nodal metastases and a poor prognosis of patients with stage I NSCLCs. Although there were a few wide CIs for the hazard ratios in Table 4, these results might be partly because of factors like differing follow-up.

These results indicate that these biological markers associated with tumour angiogenesis or metastasis are useful for detecting aggressive tumours with high metastatic potential and a poor prognosis among early-stage tumours. In contrast, regarding the methods of surgical resection among stage I NSCLCs, there was no significant difference in the 5-year survival rates between patients treated with a lobectomy and patients treated with a segmentectomy or a wedge resection $(75.3$ vs $69.0 \%, P=0.18)$. Therefore, postoperative adjuvant chemotherapy might be necessary even in patients with stage I NSCLCs (Arriagada et al, 2004; Kato et al, 2004; Strauss et al, 2004; Winton et al, 2004), when tumours are hypervascular or have reduced expressions of metastatic suppressor genes.

On the other hand, these biological markers, including tumour angiogenesis, VEGF-A, VEGF-C, and E-cadherin expression, did not affect the prognosis of patients with stage II-III NSCLCs in the present study. Reductions of MRP-1/CD9 and KAI1/CD82 were also reported to not be correlated with the survival of locally advanced stage patients (Adachi et al, 1998). Thus, tumour angiogenesis and tumour metastatic suppressor genes are not clinical indicators for patients with locally advanced NSCLCs. These results urged us to perform the present study.

We evaluated the tumour proliferation rate using the Ki-67 proliferation index (Scagliotti et al, 1993). Ki-67 antibody recognises the nuclear antigen expressed during G1, S, G2, and $M$ phases of the cell cycle and not during the resting (G0) phase. The present study has demonstrated that the Ki-67 proliferation index is one of the significant prognostic factors in patients with stage II-III NSCLCs. In contrast, the Ki-67 proliferation index was not associated with the prognosis of stage I patients.

Regarding TS, its expression is regulated by a polymorphic tandem repeat sequence in the $5^{\prime}$-terminal regulatory region of TS gene (Horie et al, 1995), and it is also regulated by several oncogenes and tumour suppressor genes, including E2F1, retinoblastoma, and p16/INK4 (DeGregori et al, 1995; Omura et al, 2000; Angus et al, 2002). Thymidylate synthase plays a central role in the biosynthesis of thymidylate, an essential precursor for DNA synthesis. Recent studies have revealed that TS exhibits oncogene-like activity. Its expression is associated with tumour cell proliferation (Navalgund et al, 1980), as demonstrated in the 

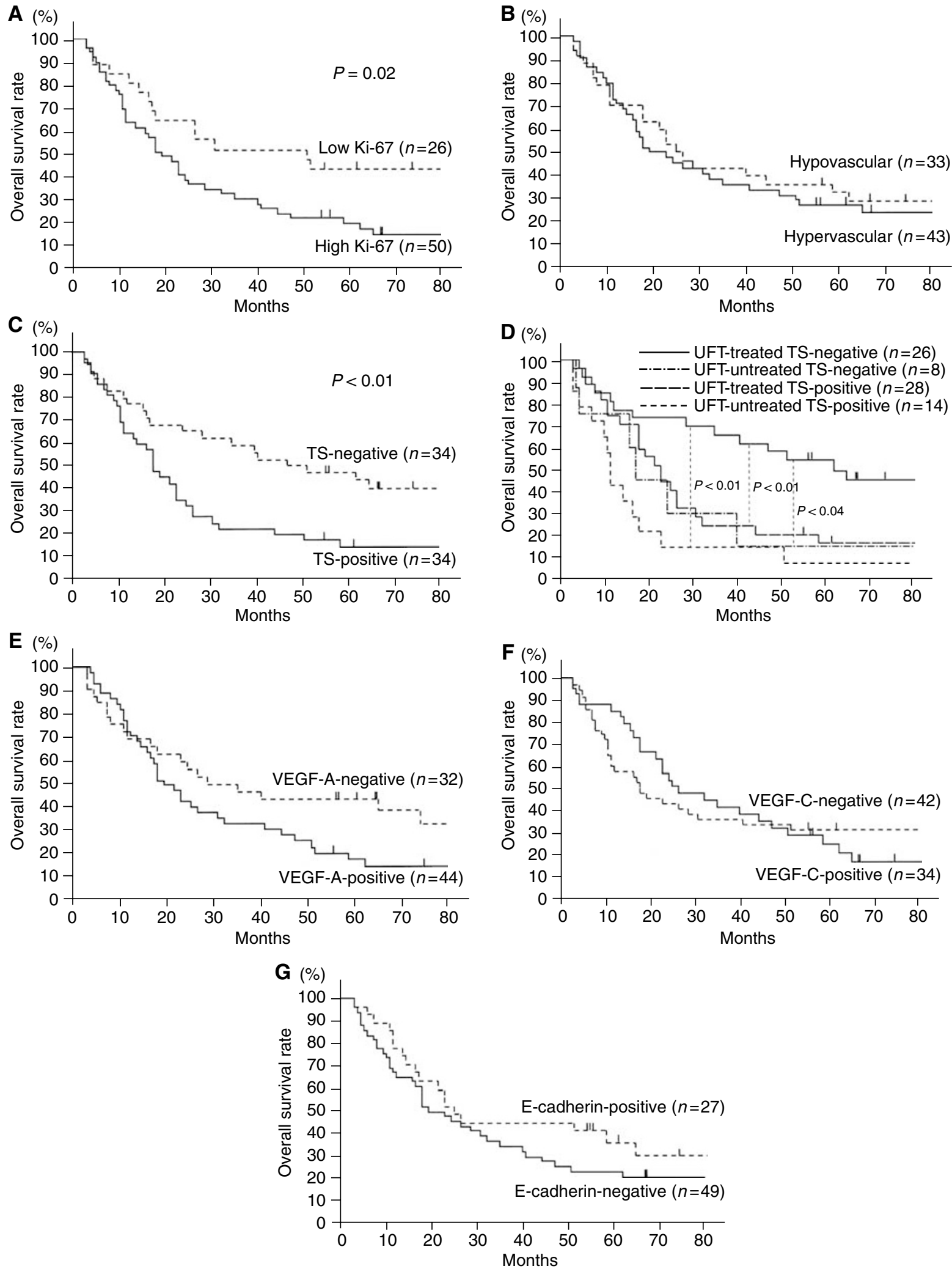

Figure 2 Overall survival of patients with stage II-III NSCLCs in relation to biological markers. (A) Ki-67 index, (B) tumour vascularity, (C) TS status, (D TS status and UFT-based chemotherapy, (E) VEGF-A status, (F) VEGF-C status, and (G) E-cadherin status.

present study. Thymidylate synthase protein also downregulates p53 expression through TS protein - p53 mRNA interaction (Chu et al, 1999). In addition, TS can induce a transformed phenotype in mammalian cells (Rahman et al, 2004). The present study has demonstrated that the intratumoral TS expression is one of significant prognostic factors in patients with stage II - III NSCLCs.

Furthermore, the intratumoral TS activity has been reported to be related to 5-FU sensitivity (Beck et al, 1994). Several clinical 
studies have demonstrated that long-term oral UFT is effective postoperative adjuvant chemotherapy for NSCLC, especially in terms of patient quality of life (Wada et al, 1996; Kato et al, 2004). Many clinical studies on human cancers, including NSCLCs and gastrointestinal tumours, have shown that high TS expression is associated with 5-FU resistance and a poor outcome (Johnston et al, 1995; Yeh et al, 1998; Huang et al, 2000). In the present study, the 5-year survival rate of UFT-treated patients with TS-negative tumours was $53.8 \%$, even in stage II-III, which was significantly higher than those of any other group $(7.1-15.2 \%)$. A recent study has demonstrated that adjuvant chemotherapy with UFT improves the survival among patients with stage I adenocarcinoma of the lung (Kato et al, 2004). In the present study, however, there was no difference in the 5-year survival rates according to the intratumoral TS status and UFT-based chemotherapy among stage I NSCLCs or among stage I adenocarcinomas. These results might be partly due to the small number of patients studied.

These results suggest that the tumour proliferation rate and TS status are useful markers for identifying less aggressive tumours in locally advanced NSCLCs, and that TS expression is also a useful marker to evaluate responsiveness of UFT-based chemotherapy for these tumours. To our knowledge, there were few clinical reports to identify biological markers useful for the treatment of patients with locally advanced NSCLCs.
These findings indicate that evaluations of these markers, including the tumour proliferation index and target molecules for chemotherapy, would improve the clinical outcome of patients with locally advanced NSCLCs. Other than TS for UFT, epithelial growth factor receptor for Gefitinib (Mendelsohn and Baselga, 2003) and topoisomerase II for etoposide (Dingemans et al, 1999) might be useful target markers for chemotherapy in advanced NSCLC patients. In addition, such targeted therapies may lead to the development of made-to-order medicine, a new therapeutic strategy for cancer (Rosell et al, 2003; Vokes and Choy, 2003). Regarding target therapies, the present study was a retrospective clinical study with some limitations in its analysis. A further prospective randomised clinical study based on an evaluation of such biological markers, as the NCIC BR.19 study on adjuvant Gefitinib therapy (National Cancer Institute Central IRB protocol, 2002), should be performed.

\section{ACKNOWLEDGEMENTS}

We thank Dr Masakazu Fukushima (Taiho Pharmaceutical Co., Tokyo, Japan) for his kind gift of a rabbit polyclonal antibody for TS.

\section{REFERENCES}

Adachi M, Taki T, Konishi T, Huang C, Higashiyama M, Miyake M (1998) Novel staging protocol for non-small-cell lung cancers according to MRP-1/CD9 and KAI1/CD82 gene expression. J Clin Oncol 16: 1397- 1406

Angus SP, Wheeler LJ, Ranmal SA, Zhang X, Markey MP, Mathews CK, Knudsen ES (2002) Retinoblastoma tumour suppressor targets dNTP metabolism to regulate DNA replication. J Biol Chem 277: 44376-44384

Arinaga M, Noguchi T, Takeno S, Chujo M, Miura T, Uchida Y (2003) Clinical significance of vascular endothelial growth factor $\mathrm{C}$ and vascular endothelial growth factor receptor 3 in patients with nonsmall cell lung carcinoma. Cancer 97: 457-464

Arriagada R, Bergman B, Dunant A, Le Chevalier T, Pignon JP, Vansteenkiste J, International adjuvant lung cancer trial collaborative group (2004) Cisplatin-based adjuvant chemotherapy in patients with completely resected non-small-cell lung cancer. $N$ Engl J Med 350: $351-360$

Beck A, Etienne MC, Cheradame S, Fischel JL, Formento P, Renee N, Milano G (1994) A role for dihydropyrimidine dehydrogenase and thymidylate synthase in tumour sensitivity to fluorouracil. Eur J Cancer 30A: $1517-1522$

Cersosimo RJ (2002) Lung cancer: a review. Am J Health-Syst Ph 59: $611-642$

Chu E, Copur SM, Ju J, Chen T, Khleif S, Voeller DM, Mizunuma N, Patel M, Maley GF, Maley F, Allegra CJ (1999) Thymidylate synthase protein and p53 mRNA form an in vivo ribonucleoprotein complex. Mol Cell Biol 19: $1582-1594$

Cox DR (1972) Regression models and life-tables. J R Stat Soc Ser B 34: $187-202$

DeGregori J, Kowalik T, Nevins JR (1995) Cellular targets for activation by the E2F1 transcriptional factor include DNA synthesis- and G1/Sregulatory genes. Mol Cell Biol 15: 4215-4224

Dingemans AC, Witlox MA, Stallaert RA, van der Valk P, Postmus PE, Giaccone G (1999) Expression of DNA topoisomerase II $\alpha$ and topoisomerase II $\beta$ genes predicts survival and response to chemotherapy in patients with small cell lung cancer. Clin Cancer Res 5: 2048-2058

Dvorak HF, Brown LF, Detmar M, Dvorak AM (1995) Vascular permeability factor/vascular endothelial growth factor, microvascular hyperpermeability, and angiogenesis. Am J Pathol 146: 1029-1039

Folkman J (1995) Angiogenesis in cancer, vascular, rheumatoid and other disease. Nat Med 1: 27-31

Gerdes J, Lemke H, Baisch H, Wacker HH, Schwab U, Stein H (1984) Cell cycle analysis of a cell proliferation-associated human nuclear antigen defined by the monoclonal antibody Ki-67. J Immunol 133: $1710-1715$
Grambsch PM, Therneau TM (1994) Proportional hazards tests and diagnostics based on weighted residuals. Biometrika 81: 515-526

Horie N, Aiba H, Oguro K, Hojo H, Takeishi K (1995) Functional analysis and DNA polymorphism of the tandemly repeated sequences in the $5^{\prime}$ terminal regulatory region of the human gene for thymidylate synthase. Cell Struct Funct 20: $191-197$

Huang C, Taki T, Adachi M, Konishi T, Higashiyama M, Kinoshita M, Hadama T, Miyake M (1998) Mutations of p53 and K-ras genes as prognostic factors for non-small cell lung cancer. Int J Oncol 12: 553 - 563

Huang C, Yokomise H, Kobayashi S, Fukushima M, Hitomi S, Wada H (2000) Intratumoral expression of thymidylate synthase and dihydropyrimidine dehydrogenase in non-small cell lung cancer patients treated with 5-FU-based chemotherapy. Int J Oncol 17: 47-54

Imoto H, Osaki T, Taga S, Ohgami A, Ichiyoshi Y, Yasumoto K (1998) Vascular endothelial growth factor expression in non-small-cell lung cancer: prognostic significance in squamous cell carcinoma. J Thorac Cardiovasc Surg 115: 1007 - 1014

Johnston PG, Lenz H, Leichman CG, Danenberg KD, Allegra CJ, Danenberg PV, Leichman L (1995) Thymidylate synthase gene and protein expression correlate and are associated with response to 5-fluorouracil in human colorectal and gastric tumors. Cancer Res 55: 1407-1412

Kajita T, Ohta Y, Kimura K, Tamura M, Tanaka Y, Tsunezuka Y, Oda M, Sasaki T, Watanabe G (2001) The expression of vascular endothelial growth factor $\mathrm{C}$ and its receptors in non-small cell lung cancer. $\mathrm{Br} J$ Cancer 85: $255-260$

Kato H, Ichinose Y, Ohta M, Hata E, Tsubota N, Tada H, Watanabe Y, Wada H, Tsuboi M, Hamajima N, Ohta M, Japan Lung Cancer Research Group on Postsurgical Adjuvant Chemotherapy (2004) A randomized trial of adjuvant chemotherapy with uracil-tegafur for adenocarcinoma of the lung. $N$ Engl J Med 350: 1713-1721

Liu D, Huang C, Kameyama K, Hayashi E, Yamauchi A, Kobayashi S, Yokomise H (2001) E-cadherin expression associated with differentiation and prognosis in patients with non-small cell lung cancer. Ann Thorac Surg 71: $949-955$

Machtay M, Lee JH, Stevenson JP, Shrager JB, Algazy KM, Treat J, Kaiser LR (2004) Two commonly used neoadjuvant chemotherapy regimens for locally advanced stage III non-small cell lung carcinoma: long-term results and associations with pathologic response. J Thorac Cardiovasc Surg 127: $108-113$

Martin J, Ginsberg RJ, Venkatraman ES, Bains MS, Downey RJ, Korst RJ, Kris MG, Rusch VW (2002) Long-term results of combined-modality therapy in resectable non-small-cell lung cancer. J Clin Oncol 20: 1989-1995 
Masuya D, Huang C, Liu D, Kameyama K, Hayashi E, Yamauchi A, Kobayashi S, Haba R, Yokomise H (2001) The intratumoral expression of vascular endothelial growth factor and interleukin-8 associated with angiogenesis in nonsmall cell lung carcinoma patients. Cancer 92: $2628-2638$

Matsuyama K, Chiba Y, Sasaki M, Tanaka H, Muraoka R, Tanigawa N (1998) Tumor angiogenesis as a prognostic marker in operable nonsmall cell lung cancer. Ann Thorac Surg 65: 1405-1409

Mbalaviele G, Dunstan CR, Sasaki A, Williams PJ, Mundy GR, Yoneda T (1996) E-cadherin expression in human breast cancer cells suppresses the development of osteolytic bone metastases in an experimental metastasis model. Cancer Res 56: $4063-4070$

McCarty Jr KS, Szabo E, Flowers JL, Cox EB, Leight GS, Miller L, Konrath J, Soper JT, Budwit DA, Creasman WT, Seigler HF, McCarty Sr KS (1986) Use of a monoclonal anti-estrogen receptor antibody in the immunohistochemical evaluation of human tumors. Cancer Res 46: 4244s-4248s

Mendelsohn J, Baselga J (2003) Status of epidermal growth factor receptor antagonists in the biology and treatment of cancer. J Clin Oncol 21: $2787-2799$

Mountain CF (1997) Revisions in the international system for staging lung cancer. Chest 111: $1710-1717$

Nakashima T, Huang C, Liu D, Kameyama K, Masuya D, Ueno M, Haba R, Yokomise H (2004) Expression of vascular endothelial growth factor-A and vascular endothelial growth factor-C as prognostic factors for nonsmall cell lung cancer. Med Sci Monit 10: 157-165

National Cancer Institute Central IRB protocol (2002) NCIC BR.19. A phase III prospective randomized, double-blind, placebo-controlled trial of the epidermal growth factor receptor antagonist, ZD1839 (Iressa) in completely resected primary stage IB, II and IIIA non-small cell lung cancer. http://www.ncicirb.org/CIRB_Protocols.asp

Navalgund LG, Rossana C, Muench AJ, Johnson LF (1980) Cell cycle regulation of thymidylate synthetase gene expression in cultured mouse fibroblasts. J Biol Chem 255: 7386-7390

Omura K, Uno Y, Kawakami K, Kanehira E, Tawaraya K, Tsukayama M, Hiranuma C, Watanabe Y (2000) Expression of thymidylate synthase in human gastric and colorectal adenocarcinomas is upregulated by $\mathrm{p} 16 /$ INK4. Hepatogastroenterology 47: $742-745$
Rahman L, Voeller D, Rahman M, Lipkowitz S, Allegra C, Barrett JC, Kaye FJ, Zajac-Kaye M (2004) Thymidylate synthase as an oncogene: a novel role for an essential DNA synthesis enzyme. Cancer Cell 5: 341-351

Rosell R, Taron M, Alberola V, Massuti B, Felip E (2003) Genetic testing for chemotherapy in non-small cell lung cancer. Lung Cancer 41: S97-S102

Scagliotti GV, Micela M, Gubetta L, Leonardo E, Cappia S, Borasio P, Pozzi E (1993) Prognostic significance of Ki67 labelling in resected non small cell lung cancer. Eur J Cancer 29A: 363-365

Skobe M, Hawighorst T, Jackson DG, Prevo R, Janes L, Velasco P, Riccardi L, Alitalo K, Claffey K, Detmar M (2001) Induction of tumor lymphangiogenesis by VEGF-C promotes breast cancer metastasis. Nat Med 7: $192-198$

Strauss GM, Herndon J, Maddaus MA, Johnstone DW, Johnson EA, Watson DM, Sugarbaker DJ, Schilsky RL, Green MR (2004) Randomized clinical trial of adjuvant chemotherapy with paclitaxel and carboplatin following resection in stage IB non-small cell lung cancer (NSCLC): report of cancer and leukemia group B (CALGB) protocol 9633. Proc Am Soc Clin Oncol 22: 621 (abstract 7019)

Sulzer MA, Leers MP, van Noord JA, Bollen EC, Theunissen PH (1998) Reduced E-cadherin expression is associated with increased lymph node metastasis and unfavorable prognosis in non-small cell lung cancer. $A m J$ Respir Crit Care Med 157: 1319-1323

Vokes EE, Choy H (2003) Targeted therapies for stage III non-small cell lung cancer: integration in the combined modality setting. Lung Cancer 41: $\mathrm{S} 115-\mathrm{S} 121$

Wada H, Hitomi S, Teramatsu T (1996) Adjuvant chemotherapy after complete resection in non-small-cell lung cancer. J Clin Oncol 14: $1048-1054$

Winton TL, Livingston R, Johnson D, Rigas J, Cormier Y, Butts C, Ding K, Seymour L, Magoski N, Shepherd F (2004) A prospective randomised trial of adjuvant vinorelbine (VIN) and cisplatin (CIS) in completely resected stage IB and II non small cell lung cancer (NSCLC) Intergroup JBR. 10. Proc Am Soc Clin Oncol 22: 621 (abstract 7018)

Yeh K, Shun C, Chen C, Lin J, Lee W, Lee P, Chen Y, Cheng A (1998) High expression of thymidylate synthase is associated with the drug resistance of gastric carcinoma to high dose 5-fluorouracil-based systemic chemotherapy. Cancer 82: 1626-1631 